\title{
Efficient Multicasting Scheme for Irregular Mesh-Based NoCs
}

\author{
Xiaohang Wang ${ }^{1,2}$, Mei Yang ${ }^{2}$, Yingtao Jiang ${ }^{2}$, Peng Liu ${ }^{1}$ \\ ${ }^{1}$ Dept. of Information Science and Electronic Engineering, Zhejiang University, P. R. China, 310027 \\ ${ }^{2}$ Dept. of Electrical and Computer Engineering, University of Nevada, Las Vegas, USA, 89154 \\ Emails: baikeina@yahoo.com.cn, Mei.Yang@unlv.edu, yingtao@egr.unlv.edu, liupeng@isee.zju.edu.cn
}

\begin{abstract}
In this paper, a simple, yet efficient hardwarebased multicasting scheme is proposed for irregular mesh-based Network-on-Chips. First, an irregular oriented multicast strategy is proposed. Literally, following this strategy, an irregular oriented multicast routing algorithm can be designed based on any regular mesh-based multicast routing algorithm. One such algorithm, namely, Alternative $X Y(A L+X Y)$, is proposed based on $X Y$ routing, Experimental results show that $A L+X Y$ achieve significant reduction in power consumption and packet latency compared with existing solutions.
\end{abstract}

\section{INTRODUCTION}

With the development of diverse applications and programming models on Chip Multiprocessors (CMPs), one-to-many communication and one-toall communication are becoming more common. Efficient support of one-to-many communications in CMPs, particularly hardware multicast support, will benefit a wide range of applications by boosting the network performance with reduced power consumption. Unfortunately, up to date, there is only very limited number of chip router designs that actually support multicasting $[1,2]$.

In addition, the following issues make multicast supporting even more complicated. The first issue is topology irregularity. The irregular sub-network and traffic isolation requirements together negate regular $2 \mathrm{D}$ mesh oriented routing algorithms, like $X Y$ routing, odd-even routing, etc. The second issue is unpredictability of the application communication behavior. Different types of applications, such as desktop, server, embedded systems, will be executed on general purpose CMPs. It is impossible to pre-characterize the communication patterns among the cores inside a sub-network. As a result, customized NoC routing approaches (like the ones using routing tables) may not be feasible.

Several NoC based multicast approaches have been proposed for NoCs with regular mesh topology. The virtual circuit tree based multicast (VCTM) [1] avoids sending redundant packets as multiple unicast. However, it uses a lookup table based multicasting router which has high power and area overhead. The approaches in [3] extend the unicast $X Y$ routing to support multicasting. The approach in [3] is referred as multicast $X Y$ in later text. In [5], tree-based adaptive multicast routing approaches are proposed. The region partition multicast (RPM) [1] selects the replication points for multicast packets based on the distribution of destinations in the network partition.

However, the aforementioned approaches [1, 2] $[3,5]$ cannot support multicasting for irregular subnetworks. The bLBDR routing [4] proposed for collective communication in irregular sub-networks supports multicasting by broadcasting in subnetworks. In bLBDR, connectivity bits are used to define different sub-networks. However, the broadcast nature of this scheme makes the network congested and results in higher power consumption.

In this paper, an irregular sub-network oriented multicast strategy is first proposed. Following this strategy, an irregular sub-network oriented multicast routing algorithm, namely, alternative $X Y$ Multicast $(A L+X Y)$, is developed based on multicasting $X Y$ [6], an efficient multicast routing algorithm proposed for regular mesh topology. To our best knowledge, our approach is the first multicast routing approach, as opposed to the broadcast-based one [4], that targets to irregular sub-networks.

\section{PRELIMINARIES}

\section{A. Architecture and Power Models}


The target NoC architecture is a tile based $\mathrm{NoC}$, which is the same as in [7]. To support multicast, the replication unit is used to at each input port to replicate flits of a multicast packet according to the decision of the routing unit. Asynchronous replication [5] scheme is chosen as the replication approach. In asynchronous replication, multiple replicated flits are allowed to be forwarded independently. If one replicated flit is blocked, other replicated flits can be forwarded asynchronously.

The power model used in [7] is followed in this study. The average power consumption for a unicast communication which sends BW bits from source tile $s$ to destination tile $t$ can be represented as,

$$
E_{\text {Unicast }}^{s, t}=\eta_{\text {hops }} \times E_{\text {Sbit }}+\left(\eta_{\text {hops }}-1\right) \times E_{\text {Lbit }}
$$

where $\eta_{\text {hops }}$ is the number of routers traversed from tile $s$ to tile $t, E_{S b i t}$ is the power consumed by the switch, and $E_{\text {Lbit }}$ is the power consumed on each link.

The average power consumption for a multicast communication which sends 1 bit from the source tile $s$ to the set of destination tiles $\bar{D}$ can be represented as,

$$
E_{\text {Multicast }}^{s, \bar{D}}=\eta_{l} \times E_{\text {Lbit }}+\eta_{R} \times E_{\text {Sbit }}
$$

where $\eta_{R}$ is the total number of routers and $\eta_{l}$ is the total number of links that are on the multicast path from tile $s$ to all tiles in $\bar{D}$, respectively.

\section{B. Assumptions and Definitions}

The following assumptions are made throughout the paper.

Assumption 1 The shape of the sub-network mapped with an application is near convex [8]. More specifically, we only consider such subnetworks that, there exists at least one minimal path (measured in hop counts) completely inside the sub-network for each pair of nodes inside the sub-network. Tiles can belong to different subnetworks.

To describe the shape of sub-networks, the following definitions are used.

Definition 1 Extended connectivity bits. Each router located at tile with coordinate $(x, y)$ has $4 \times M$ connectivity bits, $\left\{C_{N[1]}, \ldots, C_{N[M]}\right\},\left\{C_{W[1]}, \ldots, C_{W M]}\right\}$, $\left\{C_{E[1]}, \ldots, C_{E[M]}\right\}$, and $\left\{C_{S[1]}, \ldots, C_{S[M]}\right\}$. Suppose a tile has coordinate $(x, y), C_{x[q]}(q=1, \ldots, M)=1$ if tile $(x, y)$ and its neighbour tile on the $x$ direction are in the same sub-network with ID $q$. Extended connectivity (EC) bits $E C_{N}, E C_{E}, E C_{S}$, and $E C_{W}$ are defined as follows. Given the sub-network ID $q, E C_{N}=C_{N[q]}$, $E C_{W}=C_{W[q]}, E C_{E}=C_{E[q]}, E C_{S}=C_{S[q] \text {. }}$

Also the concept of regions in [1] is adopted.

\section{IRREGULAR SUB-NETWORK ORIENTED MULTICAST ROUTING}

\section{A. Motivation example and irregular sub- network oriented multicasting strategy}

Before the proposed algorithms are described in detail, an example is given to explain the motivation. Fig. 1 shows an irregular sub-network composed of 5 nodes. A multicast packet is sent from the source node to two destination nodes. The dashed line represents the path if $X Y$ multicasting is used. However, since the subnetwork is irregular, the dashed path cannot reach the destinations, i.e., at node 4 , the packet cannot go West as the link to West is not available in this sub-network.

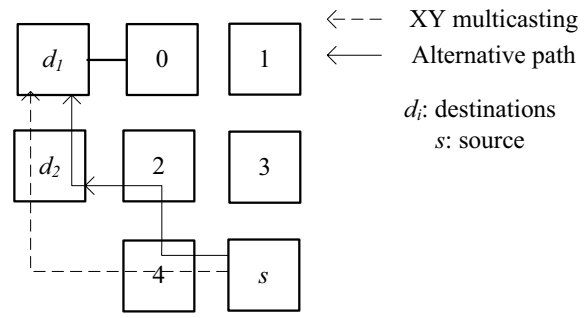

Figure 1 Path generated by $\mathrm{XY}$ and alterative path in a irregular sub-network.

Alternatively, if the packet can go North at node 4 as indicated by the solid arrow in Fig. 1, the packet can arrive at both destinations following $X Y$ from node 2.

This example shows that, if the output port found by the multicast routing algorithm is not available in the sub-network, the packet can take an alternative output port which is also on the minimal path to the destination. Each router can check the connectivity bits to see whether an output port is available. The irregular sub-network oriented multicast strategy is thus derived below.

- Find the output directions to all the destinations in the destination set using a multicast routing algorithm designed for regular mesh topology. 
- For each output direction, check the corresponding connectivity bit. If it is set, then the packet will be replicated and sent to the output direction; otherwise, use an alternative output direction.

Note that, following the strategy, an irregular sub-network oriented multicast routing algorithm can be developed based on any regular mesh oriented multicast routing algorithm. Due to the superiority of $X Y$ over other algorithms (as reviewed in Section 2), we develop Alternative $X Y$ as described below.

\section{B. Hardware-based multicast routing algorithm for irregular sub-networks}

The pseudo code of $A L+X Y$ is listed in Fig. 2.

// Alternative multicast $\mathrm{XY}$ for irregular sub-networks

// Temporary bit vectors $N_{-}$DestSet, E_DestSet, $W_{-}$DestSet $S_{-}$DestSet have the same length as IN_R $\mathrm{R}_{0}$ and are initialized to 0

// Boolean variable IN_Ri $\left(0 \leq \leq_{i} \leq 7\right)$ is 1 if region Ri includes any multicast destination nodes.

if $\left(I N \_R_{0}\right) \quad$ begin $/ /$ vector $I N \_R_{0}$ is non-zero

if $\left(E C_{E}==0\right) \quad N \_$DestSet $=I N_{-} R_{0}$ OR $N_{-}$DestSet

else $E$ D DestSet $=I N \_R_{0}$ OR E_DestSet

end

if $\left(I N_{-} R_{1}\right) \quad N_{-}$DestSet $=I N_{-} R_{1}$ OR $N_{-}$DestSet

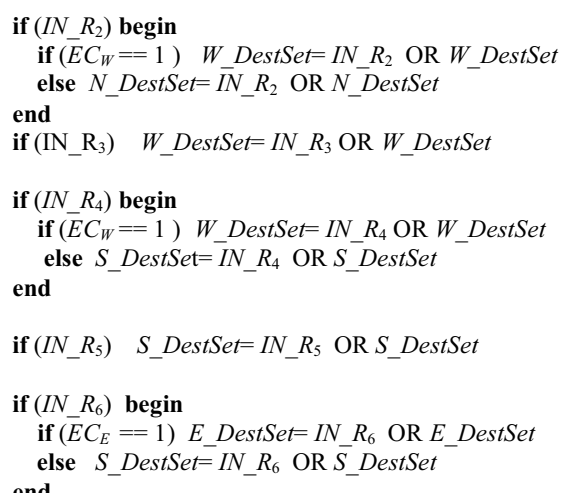

if $\left(I N \_R_{7}\right) E$ DestSet $=I N \_R_{7}$ OR $E \_$DestSet

if ( $N$ D DestSet are non-zero) replicate a packet to North with destination set $N$ DestSet // $N$ DestSet are non-zero

if $\left(E_{-}\right.$DestSet are non-zero) replicate a packet to East with destination set $E_{-}$DestSet

if ( $W$ DestSet are non-zero) replicate a packet to West with destination set $W_{-}$DestSet

if (S DestSet are non-zero) replicate a packet to South with destination set $S$ DestSet

Figure 2 Pseudocode of $A L+X Y$ multicast routing algorithm.

If multicast $X Y$ as used as the base routing algorithm, the alternative output direction is in the $Y$ direction. Thus, if there are destinations in regions 0 and 2 , North $\left(Y^{+}\right)$is selected as alternative channel. If there are destinations in regions 4 and 6 , South $(Y-)$ is selected as alternative channel. Note that, if destinations are in regions $1,3,5,7$, i.e., the $X+, X-, Y+, Y$ - regions, there is no alternative output direction. The reason is that, according to Assumption 1, the sub-network must be near convex which ensures that there exists at least a minimal path inside a sub-network for any pair of nodes. It is clear that for each destination in regions $1,3,5,7$, there is only one minimal path to that destination. Hence, there is no alternative output direction for destinations in those regions. Thus, only the alterative output directions for regions 0, 2, 4, 6 are found.

Note that the $A L+X Y$ algorithm is not deadlock free. In order to avoid deadlocks, virtual channels are used. As stated in [1], two virtual networks can be used to avoid deadlocks for mesh-based networks. For $A L+X Y$, two virtual networks are used, $V N_{0}$ and $V N_{1} . V N_{0}$ does not allow packets to turn to NORTH while $V N_{1}$ does not allow packets to turn to SOUTH. The virtual network to be used is decided for each packet at the source router and cannot be changed at the intermediate routers.

\section{Hardware cost}

Fig. 3 shows the area comparison of the baseline router and the router implementing $A L+X Y$. The baseline router supports the regular mesh topology oriented multicast $X Y$ routing algorithm [6] which uses the three types of bit vectors for four directions. The baseline router also uses two virtual networks. As shown in the table, the area overhead of $A L+X Y$ over the baseline router is $4.3 \%$. It is estimated that the percentage of the overhead tends to be stable when the network size grows.

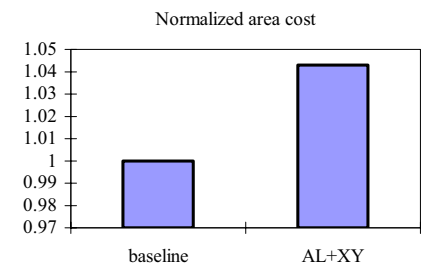

Figure 3 Area cost comparison of baseline router and the router implementing $A L+X Y$.

\section{PERFORMANCE EVALUATION}

To evaluate the performance of the $A L+X Y$ multicast routing algorithm, $A L+X Y$ is simulated 
under traces from real applications and random traffic. Due to space limit, below the simulation results under random traffic are presented. The performance of $A L+X Y$ in terms of power consumption (as defined in Section 2.1) and network latency is compared against bLBDR and multiple unicast. These multicast algorithms are implemented on the cycle accurate simulator Noxim [9]. The power parameters are based on the synthesis results using Synopses Physical compiler with TSMC 90nm library. The average bit power of routers are obtained from the synthesis results and provided to the Noxim simulator as the bit power model.

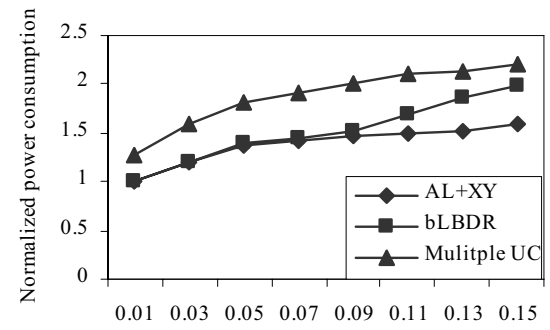

Injection rate (flit/core/cycle)

Figure 4 Normalized power consumption results with MUR set to $0.3: 1$.

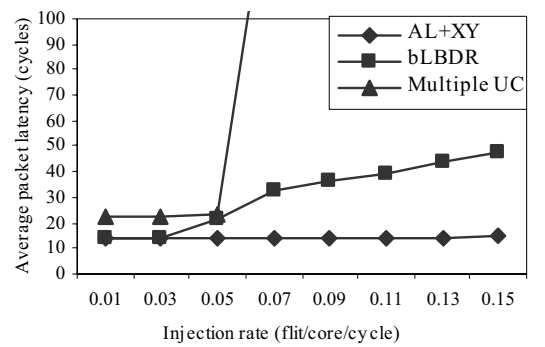

Figure 5 Latency results with MUR set to $0.3: 1$.

Fig. 4 and 5 show the results of $A L+X Y$, bLBDR, and multicast UC with the traffic ratio (MUR) of multicast (MC) to unicast (UC) traffic set to $0.3: 1$

As shown Fig. 4, $A L+X Y$ outperforms broadcast-based bLBDR and multiple unicast significantly. As the injection rate increases, the power consumption of Multiple UC and bLBDR increases much faster than that of $A L+X Y$. Multiple $\mathrm{UC}$ has the largest power consumption. For instance, in Fig. 4, AL+XY saves 29\% power consumption than that of Multiple UC. The reason is simply due to the fact that $A L+X Y$ saves a large number of replicated packets compared with multiple UC, which produces the number of replicated packets as much as the number of destinations.

$A L+X Y$ also achieve lower power consumption than bLBDR. $A L+X Y$ saves $18 \%$ power consumption than that of bLBDR. The reason is that compared with the broadcast-based bLBDR, $A L+X Y$ saves the number of replicated packets significantly, which lowers the power consumption.

In terms of average packet latency (as shown in Fig. 5), when injection rate is high (e.g., near $0.15)$, the latency of $A L+X Y$ is only $50 \%$ or less than that of bLBDR. The reason is that less packets are replicated using $A L+X Y$, thus, the network is less congested than using bLBDR and Multiple unicast. The difference becomes more distinct when MUR is larger.

The experimental results confirm that $A L+X Y$ achieve significant improvement than multiple unicast and broadcast-based approach in both power consumption and packet latency. When MUR is high and traffic is heavy, $A L+X Y$ is even superior.

\section{REFERENCES}

1. N. E. Jerger, L. S. Peh, and M. Lipasti, "Virtual circuit tree multicasting: a case for on-chip hardware multicast support," in Proc. 35th Int'l Symp. Computer Architecture, 2008, pp. 229-240.

2. L. Wang, Y. Jin, H. Kim, and E. J. Kim, "Recursive partitioning multicast: a bandwidth-efficient routing for onchip," in Proc. 3rd ACM/IEEE Int'l Symp. Networks-on-Chip 2009, pp. 64-73.

3. C. Xiang, "Design and verification of Network on chip components (in Chinese)," Master thesis, Dept. Information science and electronic engineering Zhejiang University, 2008.

4. S. Rodrigo, J. Flich, and J. Duato, "Efficient unicast and multicast support for CMPs," in Proc. 41st IEEE/ACM Int'I Symp. Microarchitecture, 2008, pp. 364-375.

5. J. Duato, S. Yalamanchili, and L. Ni, Interconnection networks an engineering approach: Morgan Kaufmann, 2002.

6. P. Abad, V. Puente, and J. A. Gregorio, "MRR: enabling fully adaptive multicast routing for CMP interconnection networks," in Proc. 15th Int'l Conf. High-Performance Computer Architecture, 2009, pp. 355-366.

7. J. Hu and R. Marculescu, "Energy-and performance-aware mapping for regular NoC architectures," IEEE Trans. Comput. Aided Design Integrated Circ. Sys., vol. 24, pp. 551-562, 2005.

8. C. L. Chou and R. Marculescu, "User-aware dynamic task allocation in networks-on-chip," in Proc. Conf. Design, automation and Test in Europe, 2008, pp. 1232-1237.

9. Noxim.[Online]. Available: www.sourceforge.net/Noxim 